4. Conclusions. This method appears to be attractive for use in the problem of calculating all eigenvalues and a complete set of orthonormalized eigenvectors for the following reasons:

(a) Like the cyclic threshold Jacobi method, there is no search for largest pivotal elements.

(b) Unlike the cyclic threshold Jacobi method there is no limitation on the angle of rotation in the Jacobi rotation as Forsythe and Henrici (1) showed was necessary for convergence.

(c) Each iteration creates $n-1$ zeros at the cost of 3 square roots as compared to one zero in the Jacobi method at the cost of 2 square roots.

Acknowledgments. The author wishes to thank Dr. W. Givens, Dr. R. Gregory and Dr. R. C. Sahni for helpful discussions in connection with this work.

New York University

New York 3, New York

1. G. E. Forsythe \& P. Henrici, "The cyclic Jacobi method for computing the principal values of a complex matrix," Trans. Amer. Math. Soc. v. 94, 1960, p. 1-23.

2. H. H. Goldstine, F. M. Murray, \& J. Von NeumanN, "The Jacobi method for symmetric matrices," J. Assoc. Comput. Mach., v. 6, 1959, p. 59-96.

3. J. H. Wilkinson, "Householder's method for the solution of the algebraic eigenvalue problem," Comput. J. v. 3, 1960, p. 23-27.

\title{
The Calculation of Certain Bessel Functions
}

\section{By D. B. Hunter}

1. Introduction. The problem of calculating Bessel functions in a digital computer has engaged the attention of a number of authors in recent years, and a variety of methods is now available. Apart from the obvious use of the power-series for small arguments and the asymptotic expansions for large arguments, the methods which have been proposed include those based on the recurrence-relations (Stegun and Abramowitz [6]; Goldstein and Thaler [3]), phase amplitude methods (Goldstein and Thaler [2]), and methods based on quadrature formulas (Fettis [1], Luke [5]).

Particular difficulties arise in the case of the modified Bessel functions of the second kind, $K_{n}(z)$ with $z$ positive; for unless $z$ is small, the power-series for $K_{n}(z)$ resolves into a difference of two large numbers which are almost equal, with a consequent loss of significant digits. The asymptotic expansion and the phase-amplitude method, on the other hand, do not yield reasonable accuracy until $|z|$ is fairly large. Thus for medium-sized values some other approach must be used. For such values the quadrature methods are convenient.

2. Quadrature Methods. The methods of Fettis [1] and Luke [5] are based on the application of numerical integration to the expression

$$
K_{\nu}(z)=\int_{0}^{\infty} e^{-z \cosh \theta} \cosh \nu \theta d \theta
$$

Received August 13, 1962. Revised July 22, 1963. 
The method used is the trapezoidal rule:

$$
\int_{0}^{\infty} F(t) d t=h\left[\frac{1}{2} F(0)+\sum_{r=1}^{\infty} F(r h)\right]-\frac{1}{2} E(h),
$$

where $h$ is some suitable interval. It is known that for a certain class of integrals $E(h)$ is remarkably small; see e.g. Fettis [1], Luke [5], and Goodwin [4].

Fettis shows that provided $h$ is suitably chosen equation (2) without the error term can be used to estimate $K_{\nu}(z)$ with high accuracy. The method can be used for any positive value of $z$. However, for a given value of $h$, the error increases with increasing $z$. Thus it is necessary to decrease $h$ progressively for large values of $z$. The object of the present paper is to deduce an alternative expression for which the error associated with a given value of $h$ decreases as $|z|$ increases. The method is applicable for non-negative integer values, $n$, of $\nu$ and general complex values of $z$.

We shall start from the alternative expression to (1);

$$
K_{n}(z)=\frac{\sqrt{\pi}\left(\frac{1}{2} z\right)^{n}}{\Gamma\left(n+\frac{1}{2}\right)} \int_{0}^{\infty} e^{-z \cosh \varphi} \sinh ^{2 n} \varphi d \varphi .
$$

(See e.g., Watson [8].) On making the substitution

$$
t=\sqrt{2 z} \sinh \frac{1}{2} \varphi
$$

it is easy to show that if $z$ is real this transforms to

$$
K_{n}(z)=\frac{\sqrt{\pi} e^{-z}}{\Gamma\left(n+\frac{1}{2}\right)(2 z)^{n}} \int_{-\infty}^{\infty} e^{-t^{2}} t^{2 n}\left(2 z+t^{2}\right)^{n-1 / 2} d t .
$$

By analytic continuation, this result is valid for $|\arg z|<\pi$. If $\arg z=\pi, K_{n}(z)$ can be calculated from the expression

$$
K_{n}\left(z e^{i \pi}\right)=e^{-n \pi i} K_{n}(z)-i \pi I_{n}(z) .
$$

$I_{n}(z)$ in this equation can be calculated from the approximations given by Fettis and Luke.

We shall estimate the error in approximating (5) by the trapezoidal rule by a method devised by Turing [7] and applied by Goodwin [4] to integrals of the form $\int_{-\infty}^{\infty} e^{-x^{2}} f(x) d x$, where $f(x)$ is even. By considering the integral of the function

$$
\frac{e^{-w^{2}} f(w)}{1-e^{-2 \pi i w / h}}
$$

of the complex variable $w$ round a rectangular contour $\Gamma$, say, with vertices at $\pm \infty \pm i a$, where $a>0$, Goodwin showed that the error $E(h)$ is given by

$$
E(h)=2 e^{a(a-2 \pi / h)} \int_{-\infty}^{\infty} \frac{e^{-t^{2}+2 i(a-\pi / h) t} f(t-i a) d t}{1-e^{-2 \pi a / h-2 \pi i t / h}} .
$$

This holds provided $f(w)$ is analytic within and on $\Gamma$.

In fact, Goodwin obtained (6) in the special case $a=\pi / h$; but, in order to avoid the branch-points of $f(w)$, we must stipulate that

$$
a<|\operatorname{Im} \sqrt{-2 z}| \text {. }
$$


It follows from (6) that

$$
|E(h)| \leqq g(a) \int_{-\infty}^{\infty} e^{-t^{2}}|f(t-i a)| d t
$$

where

$$
g(a)=\frac{2 e^{a(a-2 \pi / h)}}{1-e^{-2 \pi a / h}} .
$$

Equation (5) can alternatively be approximated by the modified trapezoidal rule of Luke [5]. The error $E^{\prime}(h)$ of this approximation can be estimated by integrating

$$
\frac{e^{-w^{2}} f(w)}{1+e^{-2 \pi i w / h}}
$$

round $\Gamma$, and is given by

$$
E^{\prime}(h)=-2 e^{a(a-2 \pi / h)} \int_{-\infty}^{\infty} \frac{e^{-t^{2}+2 i(a-\pi / h) t} f(t-i a) d t}{1+e^{-2 \pi a / h-2 \pi i t / h}} .
$$

It follows that $E^{\prime}(h)$ also satisfies inequality (8).

3. Limits on the Error. We shall now use (8) to obtain some bounds for the error $E(h)$. Those bounds will usually overestimate the error considerably, but they will be sufficiently sensitive to show that the relative error decreases as $|z| \rightarrow \infty$, and to produce a fairly simple formula for large $z$.

It is convenient to consider the cases $n=0$ and $n \geqq 1$ separately.

Case 1. $n=0$. In this case it is easy to show from (8) that

$$
|E(h)| \leqq g(a) \int_{-\infty}^{\infty} e^{-t^{2}}\left|2 x-a^{2}+t^{2}\right|^{-1 / 2} d t
$$

and this, in turn, leads to

$$
|E(h)| \leqq\left\{\begin{array}{l}
g(a) e^{x-1 / 2 a^{2}} K_{0}\left(x-\frac{1}{2} a^{2}\right) \quad \text { if } x>\frac{1}{2} a^{2} \\
g(a) e^{x-1 / 2 a^{2}}\left[K_{0}\left(\frac{1}{2} a^{2}-x\right)+\pi I_{0}\left(\frac{1}{2} a^{2}-x\right)\right] \text { if } x<\frac{1}{2} a^{2} .
\end{array}\right.
$$

If we set $a=\pi / h$, it follows from (11) and the asymptotic expansions of $I_{0}(z)$ and $K_{0}(z)$ that the relative error, $\epsilon(h)$ when $|x|$ is large satisfies

$$
|\epsilon(h)| \leqq g(\pi / h)|\sec \theta|^{1 / 2}
$$

where $\theta=\arg z$.

The above limits are quite satisfactory if $|x|$ is large compared with $|y|$. However, if $|y|$ exceeds $|x|$, a closer estimate can be obtained by subdividing the range of integration in (5) into three parts, separated by the points $t=y / 2 a$ and $t=y / a$. The result (omitting the details of the working, which are somewhat tedious) is:

$$
|E(h)| \leqq \frac{g(a)}{\sqrt{2}}\left[\sqrt{\frac{\pi}{2|y|}}+\frac{\sqrt{2|y|}}{a} e^{-y^{2} / 4 a^{2}}+\frac{\Gamma\left(\frac{1}{4}\right)}{2 \sqrt{a}} e^{-y^{2} / a^{2}}\right] .
$$

Again, we can deduce a limit on $|\epsilon(h)|$ for large $y$, namely:

$$
|\epsilon(h)| \leqq g(\pi / h)\left|\frac{1}{2} \operatorname{cosec} \theta\right|^{1 / 2}
$$


Case 2. $n \geqq 1$. Apart from the factors outside the integral in (5), the error in this case is easily shown to satisfy

$$
|E(h)| \leqq g(a) \int_{-\infty}^{\infty} e^{-t^{2}}\left(a^{2}+t^{2}\right)^{n}\left(2|z|+a^{2}+t^{2}\right)^{n-1 / 2} d t
$$

If we now fit into this the inequalities

$$
\left(a^{2}+t^{2}\right)^{\nu} \leqq\left\{\begin{array}{l}
a^{2 \nu}+t^{2 \nu} \text { if } 0 \leqq \nu \leqq 1 \\
2^{\nu-1}\left(a^{2 \nu}+t^{2 \nu}\right) \text { if } \quad \nu \geqq 1,
\end{array}\right.
$$

which hold for all values of $a$ and $t$, we obtain the result:

$$
\begin{aligned}
&|E(h)| \leqq 2^{2 n-5 / 2} g(a)\left[a^{2 n}\left(2|z|+a^{2}\right)^{n-1 / 2} \Gamma\left(\frac{1}{2}\right)+a^{2 n} \Gamma(n)\right. \\
&\left.+\left(2|z|+a^{2}\right)^{n-1 / 2} \Gamma\left(n+\frac{1}{2}\right)+\Gamma(2 n)\right] . \quad(n \geqq 2)
\end{aligned}
$$

If $n=1$, an extra factor $\sqrt{2}$ must be included in the right-hand side.

If $|z|$ is large the relative error is subject to

$$
|\epsilon(h)| \leqq 2^{2 n-5 / 2} g(\pi / h)\left[1+\frac{\Gamma\left(\frac{1}{2}\right)}{\Gamma\left(n+\frac{1}{2}\right)}\left(\frac{\pi}{h}\right)^{2 n}\right]
$$

if $n \geqq 2$. Again, an extra factor $\sqrt{2}$ must be included if $n=1$.

4. Numerical Examples. As a first example, consider the error in estimating $K_{0}(x)$ by the trapezoidal rule with $h=\frac{1}{2}$, for real positive $x$.

If $x$ is very large, inequality (12) shows that

$$
|\epsilon(h)| \leqq \frac{2 e^{-4 \pi^{2}}}{1-e^{-8 \pi^{2}}} \approx 1.431 \times 10^{-17}
$$

Even when $x$ is fairly small, it is possible to obtain quite a high degree of accuracy. For example, when $x=2$, the minimum value of the right-hand side of (11) is found to occur when $a=1.9675$ approximately, and this leads to $|E(h)| \leqq$ $5.360 \times 10^{-9}$. The corresponding relative error is subject to $|\epsilon(h)| \leqq 6.369 \times 10^{-9}$. This compares fairly well with the actual value of $\epsilon(h)$, which was found by numerical experiment to be about $9.589 \times 10^{-10}$.

The case of purely imaginary values of $z$ is interesting in view of the relation

$$
K_{n}\left(y e^{-1 / 2 \pi i}\right)=\frac{1}{2} \pi e^{1 / 2(n+1) \pi i} H_{n}{ }^{(1)}(y) .
$$

This means that by separating the result into real and imaginary parts we obtain values of the Bessel functions $J_{n}(y)$ and $Y_{n}(y)$. In his paper, Luke mentions that no integral representation of $Y_{n}(y)$ suitable for calculation by the trapezoidal rule for real values of $y$ appears to exist. Effectively, equation (5) with $z=y e^{-1 / 2 \pi i}$ provides such a representation.

The error in this case is given by inequalities (13) and (14). Consider again the variation with $y$ of the error corresponding to the value $\frac{1}{2}$ for $h$. If $|y|$ is large inequality (13) shows that

$$
|\dot{\epsilon}(h)| \leqq \frac{\sqrt{2} e^{-4 \pi^{2}}}{1-e^{-8 \pi^{2}}} \approx 1.012 \times 10^{-17}
$$


and when $y=2$ we obtain, on putting $a=\sqrt{2}$ in (13), the result $|E(h)| \leqq$ $3.835 \times 10^{-7}$, corresponding to an error of less than $2.441 \times 10^{-7}$ in $H_{0}{ }^{(1)}(y)$.

5. Alternative Method. The following alternative expression to equation (5) was suggested to the author by one of the referees:

$$
K_{n}(\lambda z)=\frac{\sqrt{\pi} e^{-\lambda z}(z / 2 \lambda)^{n}}{\Gamma\left(n+\frac{1}{2}\right)} \int_{-\infty}^{\infty} e^{-z t^{2}} t^{2 n}\left(2 \lambda+t^{2}\right)^{n-1 / 2} d t
$$

valid for $\mathrm{Rl}(z)>0$.

The error in evaluating the integral in this equation by the trapezoidal rule is readily shown, by an argument similar to Goodwin's, to be:

$$
E(h)=2 e^{a(z a-2 \pi / h)} \int_{-\infty}^{\infty} \frac{e^{-z t^{2}+2 i(z a-\pi / h) t}(t-i a)^{n}\left[2 \lambda+(t-i a)^{2}\right]^{n-1 / 2} d t}{1-e^{-2 \pi a / h-2 \pi i t / h}} .
$$

We shall not give a detailed error analysis here, but shall simply point out that, as in Fettis' method, the error increases with increasing $z$, so that the method is most suitable for small values of $z$.

6. Practical Application of the Method. The methods described above for calculating $K_{n}(z)$ are both quite convenient for programming for a digital computer. Considering first the method based on equation (5), it is clear that, for a fixed value of $h$, the values of $(r h)^{2 n} e^{-r^{2} h^{2}}$ can be held as program constants, or, alternatively, can be simply generated from the single value $h^{2 n} e^{-h^{2}}$. Then nothing more elaborate than square roots are needed in forming the terms of the approximation (2).

Similarly, in evaluating the right-hand side of (17) by the trapezoidal rule, the values of $(r h)^{2 n}\left(2 \lambda+r^{2} h^{2}\right)^{n-1 / 2}$, for fixed $\lambda$ and $h$, can be held as program constants; the values of $e^{-z r^{2} h^{2}}$ can then be generated, as before, from the single value $e^{-z h^{2}}$.

7. Acknowledgements. The author wishes to thank Dr. N. Mullineux of the College of Advanced Technology, Birmingham, England, for his helpful advice during the preparation of this paper.

In the original form of this paper, real values of $z$ only were considered in equation (5). The author is indebted to the referees for pointing out the advantages of allowing $z$ to take complex values - this extension considerably enlarges the scope of the paper. As mentioned earlier, equation (17) is also due to one of the referees.

The General Electric Company, Limited

Birmingham 6, England

Presently at Department of Mathematics

Bradford Institute of Technology

Bradford 7, Yorkshire, England.

1. H. E. Fetris, "Numerical calculation of certain definite integrals by Poisson's summation formula," $M T A C$, v. 9,1955, p. 85.

2. M. Goldstein \& R. M. Thaler, "Bessel functions for large arguments," MTAC, v. 12, 1958 , p. 18.

3. M. Goldstein \& R. M. Thaler, "Recurrence techniques for the calculation of Bessel functions," MTAC, v. 13,1959 , p. 102 . 
4. E. T. Goodwin, "The evaluation of integrals of the form $\int_{-\infty}^{\infty} e^{-x^{2}} f(x) d x$," Proc. Cambridge Philos. Soc., v. 45, 1949, p. 241.

5. Y. L. LUKE, "Simple formulas for the evaluation of some higher transcendental functions," $J$. Math. Phys., v. 34, 1956, p. 298.

6. I. A. Stegun \& M. Abramowitz, "Generation of Bessel functions on high speed computers," $M T A C$, v. 11,1957 , p. 255.

7. A. M. Turing, "A method for the calculation of the zeta-function," Proc. London Math. Soc., Ser. 2, v. 48, 1945, p. 180.

8. G. N. WATson, A Treatise on the Theory of Bessel Functions, 2nd edition, Cambridge University Press, 1944.

\section{Tables of Zeros of Cross Product Bessel Functions $J_{p}^{\prime}(\xi) Y_{p}^{\prime}(k \xi)-J_{p}^{\prime}(k \xi) Y_{p}^{\prime}(\xi)=0$}

\section{By Helmut F. Bauer}

In the calculation of frequencies for various modes of oscillations in a container comprised by a sector of the annulus between two concentric circular cylinders filled to a height $h$ with incompressible liquid, in wave guide theory and in many other applied problems involving annular or sectional cavities, the roots of the cross product of Bessel functions

$$
\Delta_{p}(\xi)=J_{p}{ }^{\prime}(k \xi) Y_{p}{ }^{\prime}(\xi)-J_{p}{ }^{\prime}(\xi) Y_{p}{ }^{\prime}(k \xi)=0, \quad p \geqq 0
$$

are of considerable interest. The functions $J_{p}(\xi)$ and $Y_{p}(\xi)$ are Bessel functions of order $p$ of the first and second kind respectively. Here $k$ is a parameter and $0 \leqq k<1$.

$$
\text { If } p=0
$$

$$
\Delta_{0}(\xi)=J_{1}(\xi) Y_{1}(k \xi)-J_{1}(k \xi) Y_{1}(\xi)=0
$$

and if $k^{\prime} \rightarrow 0$, the zeros of $\Delta_{p}(\xi)$ approach those of $J_{p}{ }^{\prime}(\xi)=0$.

J. McMahon [1] gave an asymptotic expression for the roots of equation (1). However, the lowest root was not known, until D. O. North [2] and H. Buchholz [3] mentioned its existence. Curves showing values of the roots of the expression (1) are shown in a few cases by D. Kirkham [4] for $p=0,1,2,3,4$. The purpose of this paper is to extend the range of the index $p$ and present the lowest ten roots for $p=0(1) 25, k=0(0.1) 1.0$. Bridge and Angrist [5] give the first eleven zeros for $p=0(1) 12$ and $k=1.1,1.2,1.5,(0.5) 5.0$. The present tables extend the range of $k$. The calculation of the roots was accomplished by interpolation of $\Delta_{p}(\xi)=0$.

For the calculation of the derivatives of the Bessel functions the series expanison was used in the argument range $0 \leqq x \leqq 5.0$ for the Bessel function of first kind and $0 \leqq x \leqq 5.75$ for the Bessel function of second kind. For larger arguments the asymptotic expansions were employed. For the cases in which argument and order of Bessel functions are nearly equal the results of Reference [6] have been used. The roots are correct to at least the fourth digit.

Received June 7, 1963. 